\title{
Untersuchungen über den Einbau methylierter Vorstufen in die Isoflavone der Kichererbse ${ }^{1}$
}

\author{
Wolfgang Barz * und Hans Grisebach * * \\ Lehrstuhl für Biochemie der Pflanzen der Universität Freiburg i. Br. \\ (Z. Naturforschg. 22 b, 627-633 [1967]; eingegangen am 9. Dezember 1966)
}

\begin{abstract}
In order to determine at what stage in the biosynthesis of biochanin A (5.7-dihydroxy-4'-methoxyisoflavone) and formononetin (7-hydroxy-4'-methoxyisoflavone) the methylation of the $4^{\prime}$-hydroxygroup occurs, the incorporation of $p$-methoxy-cinnamic acid- $\left[3-{ }^{14} \mathrm{C}\right.$-methyl-T], 4-methoxy-2'. $2^{\prime}$-dihydroxychalcone- $\left[\beta-{ }^{14} \mathrm{C}\right.$-methyl-T] and a number of unmethylated precursors into the isoflavones of chana seedlings (Cicer arietinum L.) was compared. $p$-Methoxy-cinnamic acid and $4.2^{\prime} .4^{\prime}$-Trihydroxychalcone were found to be the best precursors. Although the incorporation of $p$-methoxycinnamic acid was very good (incorporation rate $6.2 \%$ ) the $\mathrm{T} /{ }^{14} \mathrm{C}$ ratio dropped by $95 \%$ upon incorporation into the isoflavones, a fact which showed that a rapid demethylation takes place in the plants.

In addition, kinetic experiments were carried out with $p$-methoxycinnamic acid- $\left[3-{ }^{14} \mathrm{C}\right.$-methyl${ }^{14} \mathrm{C}$-methyl-T] and 4-methoxy-2' $2^{\prime} 4^{\prime}$-dihydroxychalcone- $\left[\beta \cdot{ }^{14} \mathrm{C}\right.$-methyl-14 $\mathrm{C}$-methyl-T]. But in this case also the rapid demethylation did not permit a definite conclusion to be drawn as to the stage in the biosynthesis at which the methylation step occurs. The very good incorporation of $4.2^{\prime} .4^{\prime}$-trihydroxycalcone- $\left[\beta-{ }^{14} \mathrm{C}\right]$ into the isoflavones $(5,3 \%$ incorporation, dilution 61$)$, however, shows that methylation can occur at a late stage in the biosynthesis.
\end{abstract}

Für die Biosynthese der in der Kichererbse (Cicer arietinum L.) vorkommenden Isoflavone Biochanin A (5.7-Dihydroxy-4'-methoxyisoflavon) und Formononetin (7-Hydrox-4'-methoxyisoflavon) sind die $(-)(2 \mathrm{~S})$ Flavanone die besten bisher aufgefundenen Vorstufen, wie wir mit $(-)(2 \mathrm{~S})$-5.7.4' Trihydroxyflavanon im Falle des Biochanin A zeigen konnten ${ }^{2}$. Da jedoch beide Isoflavone eine Methoxylruppe am C-4' besitzen, blieb die Frage offen, auf welcher Stufe die Methylierung der 4'. Hydroxylgruppe erfolgt. Zwar konnten wir eine Umwandlung von Daidzein (7.4'-Dihydroxyisoflavon) zu Formononetin in der Luzerne zeigen, jedoch war hierbei die Einbaurate sehr gering, so daß eine Methylierung auf der Stufe des Isoflavons als Hauptweg unwahrscheinlich erschien ${ }^{1}$. Hess hat den Einbau methylgruppenmarkierter Ferulasäure und Sinapinsäure in die Anthocyane von Petunia hybrida untersucht und erhielt den besten Einbau in die entsprechend methoxylierten Anthocyane ${ }^{3}$. Unbefriedigend blieb hierbei jedoch der Befund, daß nur ein Teil der Radioaktivität in der Methoxylgruppe der Anthocyane wiedergefunden wurde, so daß ein intaker Einbau der methylierten Vorstufen nicht als

1 XV. Mitt. zur Biogenese der Isoflavone. XIV. Mitt.: W. Barz u. H. Grisebach, Z. Naturforschg. 21 b, 1113 [1966].

* Gegenwärtige Anschrift: National Heart Institut, NIH Bethesda, Md., USA.

** Anschrift: Chem. Laboratorium d. Univ., 78 Freiburg i. Br., Albertstr. 21.

2 L. Patschke, W. Barz u. H. Grisebach, Z. Naturforschg. 21 b. 201 [1966].

3 D. Hess, Planta 60, 568 [1964]. bewiesen angesehen werden kann. Da auch in zellfreien Systemen die hydroxylierten Zimtsäuren die besten Substrate für die Methylierung sind, liegt der Schluß nahe, den Methylierungsschritt bereits auf der Stufe der Zimtsäuren anzunehmen ${ }^{4}$.

Wir haben daher in der vorliegenden Arbeit den Einbau von $p$-Methoxyzimtsäure, 4-Methoxy-2'.4'. dihydroxychalkon (7-Hydroxy-4'-methoxyflavanon) und nicht methylierten Vorstufen in die Isoflavone der Kichererbse verglichen. Da in Pflanzen eine Demethylierung erfolgen kann ${ }^{5,5 a}$, war für den Beweis des intakten Einbaus der methylierten Vorstufen eine Mehrfachmarkierung notwendig.

Zunächst wurden die H-Atome der jeweiligen Methoxylgruppe mit Tritium und das $\beta$-C-Atom der $p$ Methoxyzimtsäure bzw. des Chalkons mit ${ }^{14} \mathrm{C}$ markiert. Später erwies es sich jedoch als notwendig, zusätzlich das C-Atom der Methoxylgruppe mit ${ }^{14} \mathrm{C}$ zu markieren. Die Ergebnisse mit den beiden dreifach markierten Substraten zeigen, $d a ß$ in der Kichererbse eine schnelle Demethylierung erfolgt, so $\mathrm{da} ß$ mit in vivo-Versuchen nicht entschieden werden kann, auf welcher Stufe der Biosynthese der Methylierungsschritt abläuft.

4 D. Hess, Z. Naturforschg. 19 b, 447 [1964]; D. Hess, Z. Pflanzenphysiol. 53, 460 [1965]; 53, l [1965].

5 Siehe z. B. F. R. Stermitz u. H. Rapoport, J. Amer. chem. Soc. 83, 4045 [1961] ; A. R. Battersby u. B. J. T. Harper, Tetrahedron Letters [London] 27, 21 [1960].

5a Wahrscheinlich verläuft auch die bei Zimtsäuren beobachtete Demethoxylierung über eine Demethylierung mit anschließender Dehydroxylierung. Siehe z. B. S. Z. ELBasyouni, D. Chen, R. K. Ibrahim, A. C. Neish u. G. H. N. Towers, Phytochem. 3, 485 [1964]. 


\section{Ergebnisse}

Synthese der markierten Verbindungen

\section{a) 4-Methoxy-2'.4'-dihydroxychalkon- $\left[\beta-{ }^{14} C\right]$}

$p$-Hydroxybenzaldehyd-[carbonyl- ${ }^{14} \mathrm{C}$ ] ${ }^{6}$ wurde in Aceton mit $\mathrm{K}_{2} \mathrm{CO}_{3}$ und Methyljodid zu Anisaldehyd[carbonyl- ${ }^{14} \mathrm{C}$ ] methyliert und letzterer mit 4-Tetraacetyl-glucosidoresacetophenon ${ }^{7}$ kondensiert $^{7,8}$. Nach Hydrolyse des rohen Glucosids unter Stickstoff mit $\mathrm{HCl}$ wurde das Aglykon chromatographisch gereinigt.

\section{b) 4-Methoxy-2'.4'-dihydroxy-chalkon-[methyl-T] bzw. [methyl. ${ }^{14} C$ ]}

4-Hydroxybenzaldehyd wurde wie unter a) mit Methyljodid-[T] ${ }^{9}$ oder Methyljodid-[ $\left[{ }^{14} \mathrm{C}\right]$ zum $p$ Methoxybenzaldehyd-[methyl-T] bzw. [methyl- ${ }^{14} \mathrm{C}$ ] methyliert und in der oben beschriebenen Weise zum Chalkon kondensiert und nach Hydrolyse gereinigt.

\section{Pflanzenversuche}

a) Vorversuche über den Gehalt an Isoflavonglykosiden und die Verteilung der Isoflavone in der Kichererbse

Frische Kichererbsen verschiedenen Alters wurden nach grünen Teilen und Wurzeln getrennt aufgearbeitet. Die Pflanzenteile wurden im Starmix mit Aceton von $-20^{\circ} \mathrm{C}$ dreimal extrahiert und die im
Aceton enthaltenen Aglyka nach Dünnschichtchromatographie mit Benzol/Äthanol (92:8) photometrisch bestimmt. In einem Parallelversuch wurden gleiche Mengen Pflanzenteile im Starmix mit Wasser zerkleinert und der Brei 6 Stdn. bei Raumtemperatur belassen, wobei die pflanzeneigenen Glykosidasen die vorhandenen Isoflavonglykoside* vollständig spalten. Nach Absaugen der festen Teile und Trocknung eluierte man diese mit Methanol und trennte die Isoflavone wie oben durch Dünnschichtchromatographie. Die photometrische Bestimmung ergab die gesamte Isoflavon-Konzentration. Tab. 1 zeigt die Ergebnisse eines Versuches.

In mehreren Versuchen ergab sich folgendes Bild:

1. Etwa 7-20\% der Isoflavone liegt als Aglykon vor.

2. Biochanin A ist in ca. 2- bis 3-mal größerer Menge vorhanden als Formononetin.

3. Der Isoflavongehalt der Wurzeln ist etwa 2- bis 4-mal höher als derjenige der grünen Teile.

\begin{tabular}{lcc}
\hline Vor Hydrolyse & $\begin{array}{c}\text { Formononetin } \\
{[\mathrm{mg} / \mathrm{g} \text { Trockengewicht }]}\end{array}$ \\
\hline Grüne Teile & 0,94 & 0,5 \\
Wurzeln & 1,83 & 2,5 \\
Nach Hydrolyse & & \\
Grüne Teile & 3,58 & 8,5 \\
Wurzeln & 9,75 & 34,2 \\
\hline
\end{tabular}

Tab. 1. Gehalt der Kichererbsen an Isoflavonen vor und nach enzymatischer Hydrolyse.

\begin{tabular}{|c|c|c|c|c|c|c|c|}
\hline \multirow{2}{*}{ Vorstufe } & \multicolumn{3}{|c|}{ Einbaurate $[\%]$} & \multicolumn{2}{|c|}{ Verdünnung } & \multicolumn{2}{|c|}{$\mathrm{T} /{ }^{14} \mathrm{C}$} \\
\hline & Biochanin A & Form & ononetin & Biochanin A & Formononetin & Biochanin A & Formononetin \\
\hline Zimtsäure-[3-14C] & 0,51 & 3 & $(0,51)$ & 1362 & $89(158)$ & & \\
\hline$p$-Cumarsäure-[3-14C] & 0,11 & 0,74 & $(0,04)$ & 5066 & $307(431)$ & & \\
\hline$p$-Methoxyzimtsäure- & ${ }^{14} \mathrm{C}: 0,66$ & 6,2 & $(4,3)$ & 803 & $61(44)$ & $100 \rightarrow 4,46$ & $100 \rightarrow 2,85(6,96)$ \\
\hline [3-14C-Methyl-T] & $\mathrm{T}: 0,03$ & 0,18 & $(0,3)$ & 18007 & $1295(631)$ & & \\
\hline $\begin{array}{l}4.2^{\prime} .4^{\prime} \text {-Trihydroxychalkon- } \\
{[\beta-14 \mathrm{C}]}\end{array}$ & 0,006 & 5,3 & $(0,4)$ & 84440 & $61(80)$ & & \\
\hline 4-Methoxy-2'.4'-dihy- & ${ }^{14} \mathrm{C}: 0,008$ & 0,03 & $(0,01)$ & 184000 & $13360(20930)$ & $100 \rightarrow 156$ & $100 \rightarrow 71(95)$ \\
\hline $\begin{array}{l}\text { droxy-chalkon- } \\
{[\beta-14 \mathrm{C} \text {-methyl-T }]}\end{array}$ & $\mathrm{T}: 0,013$ & 0,02 & $(0,009)$ & 63480 & $10210(11811)$ & & \\
\hline Daidzein-[T] & 0,09 & 0,034 & & 71140 & 6488 & & \\
\hline
\end{tabular}

Tab. 2. Einbau verschiedener Vorstufen in die Isoflavone der Kichererbse. Die Zahlen in Klammern wurden von Formononetin erhalten, das ursprünglich in der Pflanze als Glykosid vorlag.

${ }^{6}$ H. Grisebach u. L. Patschie, Chem. Ber. 95, 2098 [1962].

7 L. Reichel u. J. Steudel, Liebigs Ann. Chem. 553, 83 [1942].

8 G. Zemplen u. R. Bognar, Chem. Ber. 95, 2098 [1962].
${ }^{9}$ F. Weygand, J. Daniel u. H. Simon, Chem. Ber. 91, 1691 [1958].

* In Kichererbsen ist bisher das Vorkommen von Biochanin A-7-glucosid nachgewiesen worden. E. Wong, P. I. Mortimer u. T. A. Geissmann, Phytochem. 4, 89 [1965]. 
b) Vergleich des Einbaus verschiedener methylierter und nicht methylierter Vorstufen in die Isoflavone

Zunächst wurden die in Tab. 2 aufgeführten markierten Verbindungen als Vorstufen für die Isoflavone bei 14 Tagen alten Cicerpflänzchen verglichen.

Jeweils 15 Pflanzen ohne Wurzeln erhielten $8 \mathrm{ccm}$ einer $3,125 \cdot 10^{-4}-m$. Lösung der markierten Verbindung $(2,5 \mu \mathrm{Mol})$ während 36 Stunden. Die nicht aufgenommene Radioaktivität wurde bei der Berechnung der Werte in Tab. 2 berücksichtigt. Um die Aktivität der Aglyka und Glykoside getrennt bestimmen zu können, extrahierte man die Pflanzen nach der Entfernung von Lipiden und Chlorophyll mit Petroläther mit Äther und gewann dann die Aglyka der zunächst als Glykoside vorliegenden Isoflavone durch Hydrolyse der wäßrigen Aufschlämmung der Pflanzenrückstände mit verd. $\mathrm{HCl}$ unter $\mathrm{Zusatz}$ von Äthanol und Extraktion der Hydrolysenlösung mit Äther. Es folgte eine im experimentellen Teil beschriebene chromatographische Reinigung. Danach wurden alle Proben nach Verdünnung mit inaktivem Material bis zur konstanten spez. Aktivität umkristallisiert.

Die Werte der Tab. 2 lassen erkennen, daß $p$ Methoxyzimtsäure- $\left[3{ }^{14} \mathrm{C}\right.$-methyl-T $]$ wohl hinsichtlich des ${ }^{14} \mathrm{C}$-Einbaus eine sehr gute Vorstufe für die Isoflavone ist, gleichzeitig aber ein sehr starker Tritiumverlust beim Einbau in die Isoflavone eintritt; d. h., es muß im Verlauf der Biosynthese eine sehr starke Demethylierung stattgefunden haben. 4-Methoxy-2 $2^{\prime} .4^{\prime}$-dihydroxychalkon- $\left[\beta-{ }^{14} \mathrm{C}\right.$-methyl-T $]$ ist dem ${ }^{14} \mathrm{C}$-Einbau nach eine bedeutend schlechtere Vorstufe, jedoch ist hier der Abfall des $\mathrm{T} /{ }^{14} \mathrm{C}$-Verhältnisses im Formononetin nur 30 Prozent. Auffallend ist aber die sehr viel geringere Spezifität des Einbaus in Formononetin im Vergleich zu Biochanin A im Gegensatz zum Versuch mit dem Trihydroxychalkon. Während mit dem Trihydroxychalkon als Vorstufe die Einbaurate in Formononetin 850-mal höher und die Verdünnung 1380-mal geringer ist als im Biochanin A, sind die entsprechenden Werte mit Methoxychalkon nur 4 und 14. Es war daher anzunehmen, daß kein intakter Einbau des Methoxychalkons in Formononetin stattfindet. Die Ergebnisse blieben auch insofern noch unbefriedigend, als lediglich ein Verlust der H-Atome der Methoxylgruppe durch die Tritiummarkierung nachgewiesen werden

* Über Versuche zur Aufnahme des Chalkons in die Pflanzen unter verschiedenen Bedingungen s. Experimenteller Teil. kann. Obwohl es unwahrscheinlich ist, daß ein TVerlust nicht durch Verlust der ganzen Methylgruppe, sondern nur durch eine Austauschreaktion zustande kommt, schien es notwendig, die Versuche mit dreifach markierten Verbindungen zu wiederholen, in denen sowohl die H-Atome wie auch das C-Atom der Methoxylgruppe und ein weiteres CAtom im Molekül markiert waren. Weiterhin erhofften wir uns durch Versuche bei kürzeren Zeiten und durch Verfolgung der zeitlichen Abhängigkeit des Einbaus eindeutigere Ergebnisse bezüglich der Beteiligung der methylierten Vorstufen bei der Biosynthese.

c) Zeitliche Verfolgung des Einbaus dreifach markierter p-Methoxyzimtsäure und dreifach markierten Methoxychalkons in die Isoflavone

Je $2,5 \mu \mathrm{Mol} p$-Methoxyzimtsäure-[3.14 C-methyl${ }^{14} \mathrm{C}$-methyl-T] bzw. 4-Methoxy-2' $.4^{\prime}$-dihydroxychalkon- $\left[\beta-{ }^{14} \mathrm{C}\right.$-methyl $-{ }^{14} \mathrm{C}$-methyl-T] gelöst in $0,3 \mathrm{~cm}$ Glykolmonomethyläther * wurden nach Zugabe von je 7,7 ccm 0,05-m. Pyrophosphatpuffer $\left(p_{\mathrm{H}} 8,5\right)$ den Pflanzen ohne Wurzeln zugeführt. Für die Parallelversuche von $8^{1 / 2}, 18$ und 26 Stdn. Dauer verwendete man je 15 Pflanzen. Im Gegensatz zu dem ersten Versuch, wurde bei der Aufarbeitung nicht zwischen Aglyka und Glykosiden differenziert, sondern sofort eine enzymatische Hydrolyse der Glykoside vorgenommen. Alle Isoflavonproben wurden nach Verdünnung mit inaktivem Material und Permethylierung durch Umkristallisieren gereinigt. Ein Abbau der Isoflavone mit Alkali zu Ameisensäure und dem entsprechendem Desoxybenzoin ${ }^{10}$ erlaubte die getrennte Aktivitätsbestimmung im C-2 und der Methoxylgruppe.

\begin{tabular}{|c|c|c|c|c|c|c|}
\hline \multirow{3}{*}{ Vorstufe } & \multicolumn{3}{|c|}{$\begin{array}{c}\text { Formononetin } \\
{[\mathrm{mg}]}\end{array}$} & \multicolumn{3}{|c|}{$\underset{[\mathrm{mg}]}{\operatorname{Biochanin} \mathrm{A}}$} \\
\hline & & Ver & achsd & aer $[S$ & dn.] & \\
\hline & $81 / 2$ & 18 & 26 & $81 / 2$ & 18 & 26 \\
\hline \multicolumn{7}{|c|}{$\begin{array}{l}p \text {-Methoxyzimtsäure- } \\
{\left[\beta-{ }^{14} \mathrm{C} \text {-methyl- }\right.}\end{array}$} \\
\hline${ }^{14} \mathrm{C}-$ methyl-T] & 4,5 & 2,4 & 3,8 & 8,4 & 6,4 & 8,6 \\
\hline $\begin{array}{l}\text { Methoxychalkon- } \\
\text { [ } \beta-{ }^{14} \mathrm{C} \text {-methyl- }\end{array}$ & & & & & & \\
\hline${ }^{14} \mathrm{C}$-methyl-T] & 4,4 & 3,4 & 2,6 & 7,9 & 8,4 & 6,4 \\
\hline
\end{tabular}

Tab. 3. Ausbeuten an Isoflavonen bei den verschiedenen Versuchen.

10 H. Grisebach, Z. Naturforschg. 14 b, 802 [1959]. 
Tab. 3 zeigt die Ausbeuten an Isoflavonen bei den verschiedenen Versuchen; diese sind relativ geringen Schwankungen unterworfen. Tab. 4 zeigt die Aufnahme der markierten Vorstufen zu verschiedenen Zeiten. Die jeweils nicht aufgenommene Aktivität wurde bei der Berechnung der Einbauraten be-

\begin{tabular}{|c|c|c|}
\hline Versuchsdauer & $\begin{array}{l}81 / 2 \text { Stdn. } 18 \text { Stdn. } \\
\text { Aktivitätsaufnahm }\end{array}$ & 26 Stdn. \\
\hline Vorstufe & ${ }^{14} \mathrm{C} \quad \mathrm{T} \quad{ }^{14} \mathrm{C} \quad \mathrm{T}$ & ${ }^{14} \mathrm{C}$ \\
\hline
\end{tabular}

$p$-Methoxyzimt-

säure-

[ $\beta$-14C-methyl-

${ }^{14} \mathrm{C}$-methyl-T]

Methoxychalkon-

$\left[\beta-{ }^{14} \mathrm{C}\right.$-methyl-

${ }^{14} \mathrm{C}$-methyl-T]

$\begin{array}{llllll}4 & 13 & 29,5 & 41 & 44,5 & 49,5\end{array}$

$26,5 \quad 15,5 \quad 41 \quad 40 \quad 51,5 \quad 47,5$

Tab. 4. Aufnahme der ${ }^{14} \mathrm{C}$ - und Tritiumaktivität durch die Pflanzen.

rücksichtigt. Der ausgeprägte Unterschied in der Aufnahme der Tritium- und ${ }^{14} \mathrm{C}$-markierten $p$ Methoxyzimtsäure ist uns nicht erklärlich. In den Abbn. 1 und 2 ist der unterschiedliche Einbau der verschiedenen Atome der Zimtsäure und des Chalkons in die Isoflavone dargestellt. Während durch die Versuche mit der dreifach markierten Zimtsäure die Ergebnisse der ersten Versuche bestätigt werden, d. h. guter Einbau des $\beta$-C, aber starke Demethylierung, wird beim Chalkonversuch unerwarteterweise das C-Atom der Methoxylgruppe weit besser als das $\beta$ - $\mathrm{C}$ in Formononetin eingebaut. Beim Biochanin A wurde hierbei nur die Summe von Methyl. ${ }^{14} \mathrm{C}+$ $\beta \cdot{ }^{14} \mathrm{C}$ erfaßt. In Ergänzung zu den Abbn. 1 und 2 sind in den Abbn. 3 und 4 die Änderung des Isotopenverhältnisses in den Isoflavonen zwischen einzelnen markierten C-Atomen dargestellt. Die Differenzierung der ${ }^{14} \mathrm{C}$-Aktivität beim Chalkonversuch durch den Abbau der Isoflavone in $\beta$-C und Methyl$\mathrm{C}$ ergibt also, daß der intakte Einbau des Chalkons in den Versuchen mit dem doppelt markierten Substrat nur vorgetäuscht wurde.

d) Gabe von p-Methoxyzimtsäure-[methyl $\left.-{ }^{14} C\right]$ und Bestimmung der Radioaktivität in der Atmungskohlensäure

$2,5 \mu \mathrm{Mol} p$-Methoxyzimtsäure-[methyl $\left.-{ }^{14} \mathrm{C}\right]$ wurden als Na-Salz an 15 Cicer-Pflanzen von 10 Tagen Alter zugeführt. Die Pflanzen befanden sich während der Versuchsdauer von 61 Stdn. in einem Exsikkator mit feuchter Atmosphäre, durch den ein leichter
Luftstrom gesaugt wurde, der nach dem Exsikkator eine Waschflasche mit Natronlauge passierte. Angeboten wurde $2,536 \cdot 10^{6} \mathrm{ipm}$; im gefällten $\mathrm{BaCO}_{3}$ fanden sich $4,97 \cdot 10^{5} \mathrm{ipm}$. Danach wurden innerhalb 61 Stdn. 20\% der von den Pflanzen aufgenommenen Radioaktivität ausgeatmet.

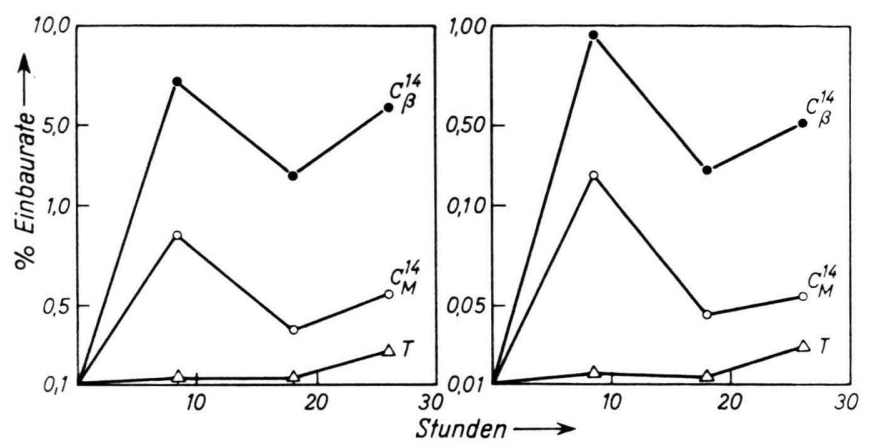

Abb. 1. Einbau der verschiedenen markierten Atome der $p$ Methoxyzimtsäure- $\left[\beta-{ }^{14} \mathrm{C}\right.$-methyl ${ }^{14} \mathrm{C}$-methyl-T] in Isoflavone in Abhängigkeit von der Versuchsdauer.

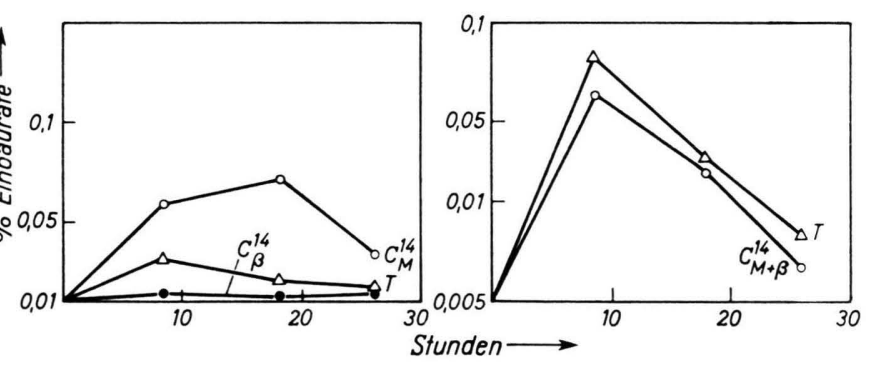

Abb. 2. Einbau der verschiedenen markierten Atome von 4-Methoxy-2'.4'-dihydroxychalkon- $\left[\beta-{ }^{14} \mathrm{C}\right.$-methyl- ${ }^{14} \mathrm{C}$-methyl-T] in Isoflavone in Abhängigkeit von der Versuchsdauer.
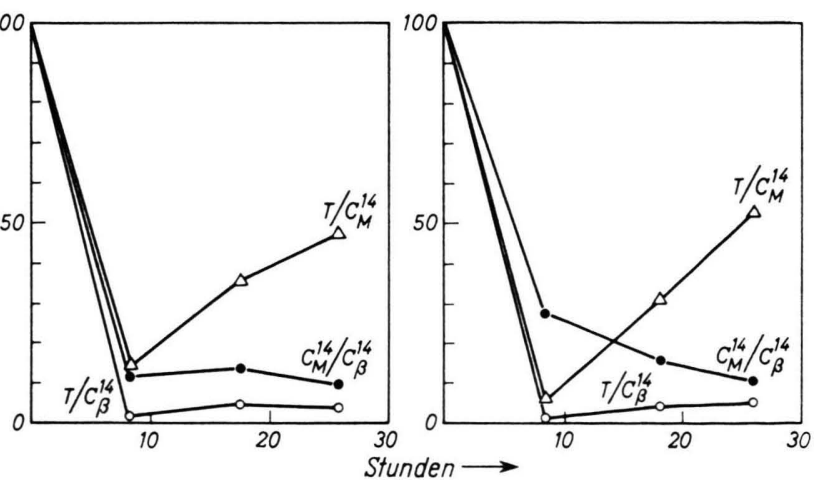

Abb. 3. Änderung des Isotopenverhältnisses vom Ausgangswert $=100$ in der Vorstufe auf den gefundenen Wert in den Isoflavonen in Abhängigkeit von der Versuchsdauer. Vorstufe: $p$-Methoxyzimtsäure- $\left[\beta-{ }^{14} \mathrm{C}\right.$-methyl $-{ }^{14} \mathrm{C}$-methyl-T] 


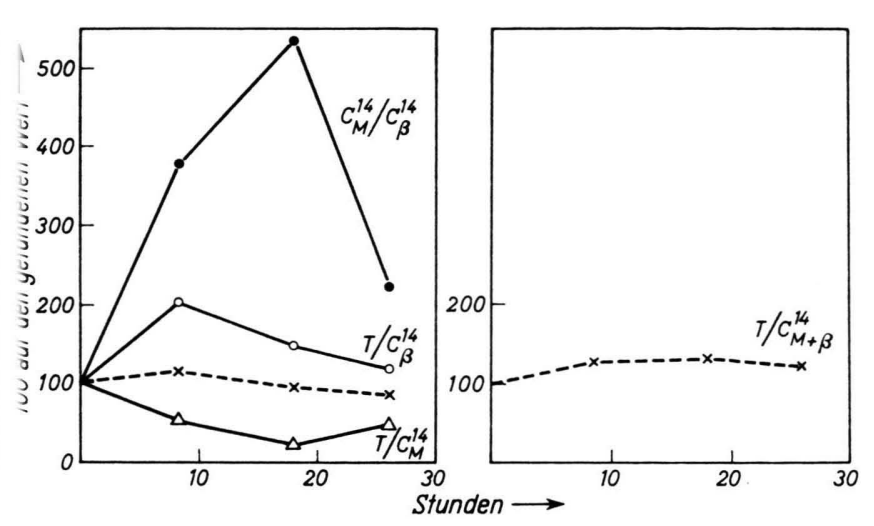

Abb. 4. Änderung des Isotopenverhältnisses vom Ausgangswert $=100$ in der Vorstufe auf den gefundenen Wert in den Isoflavonen, in Abhängigkeit von der Versuchsdauer. Vorstufe: 4-Methoxy-2'.4'-dihydroxychalkon $-\left[\beta-{ }^{14} \mathrm{C}\right.$ - methyl $-{ }^{14} \mathrm{C}$ methyl-T].

\section{Diskussion}

$p$-Methoxyzimtsäure und $4.2^{\prime} \cdot 4^{\prime}$-Trihydroxychalkon sind von den untersuchten Verbindungen die besten Vorstufen für Formononetin (Tab. 2). In Ubereinstimmung mit früheren Ergebnissen ${ }^{11} \mathrm{er}$ folgt der Einbau des Chalkons spezifisch nur in Formononetin (gleiches Hydroxylierungsmuster). Der starke Abfall des T/ $/{ }^{14} \mathrm{C}$-Verhältnisses beim Einbau der Zimtsäure in die Isoflavone zeigt, daß trotz der hohen Einbaurate und des niedrigen Verdünnungswertes eine starke Demethylierung im Verlauf der Biosynthese der Isoflavone aus der $p$-Methoxyzimtsäure eingetreten ist * ${ }^{*} \mathrm{Ob}$ diese Demethylierung bereits auf der Stufe der Zimtsäure oder später erfolgt, läßt sich nicht sagen. Der bedeutend bessere Einbau der $p$-Methoxyzimtsäure im Vergleich zu $p$ Hydroxyzimtsäure ( $p$-Cumarsäure) ist daher wohl auf die größere Beständigkeit ersterer Säure und auf einen evtl. besseren Transport innerhalb der Pflanze zurückzuführen. Im Gegensatz zum Trihydroxychalkon ist 4-Methoxy-2'.4'-dihydroxychalkon eine sehr viel schlechtere Vorstufe für Formononetin. Dies könnte zunächst auf die sehr schlechte Wasserlös-

11 L. Patschke, W. Barz u. H. Grisebach, Z. Naturforschg. $19 \mathrm{~b}, 1110$ [1964] und frühere Mitteilungen.

* Anm. b. d. Korr.: Um eine Demethylierung durch Bakterien in der zugeführten Lösung nachzuprüfen, wurden 10 Cicerpflanzen in die Lösung von $1,25 \mu \mathrm{Mol}(1,8 \mu \mathrm{C})$ $p$-Methoxyzimtsäure- $\left[3{ }^{14} \mathrm{C}\right]$ in $8 \mathrm{~cm}^{3}$ 0,05-m. Pyrophosphatpuffer $\left(p_{\mathrm{H}} 7,5\right)$ gestellt und nach $9 \mathrm{Stdn}$. in der Lösung eine Verdünnungsanalyse auf $p$-Cumarsäure durchgeführt. In der $p$-Cumarsäure fand sich keine Radioaktivität. lichkeit des Methoxychalkons zurückgeführt werden. Beim Lösen des Chalkons in Glykolmonomethyläther unter Zusatz von Pyrophosphatpuffer wurden zwar 96\% innerhalb von 36 Stdn. von den Pflanzen aufgenommen, jedoch könnte das Chalkon nach Aufnahme in die Pflanze wieder ausfallen, bevor es an den Syntheseort der Isoflavone gelangt. Andererseits zeigt ein Vergleich der ${ }^{14} \mathrm{C}$ - und T-Aktivitäten von Formononetin und Biochanin $\mathrm{A}$, daß die $\mathrm{T}$ Aktivität nicht spezifisch in Formononetin eingebaut wird und auch der Unterschied der ${ }^{14} \mathrm{C}$-Aktivitäten lange nicht so ausgeprägt ist wie mit dem Trihydroxychalkon als Vorstufe. Obwohl sich das $\mathrm{T} /{ }^{14} \mathrm{C}$-Verhältnis in diesem Versuch lange nicht so stark änderte, wie beim Versuch mit $p$-Methoxyzimtsäure, muß der Einbau des Tritiums aus den eben angeführten Gründen hauptsächlich nur auf indirektem Wege erfolgen. Den gleichen Schluß muß man beim Versuch mit Daidzein-[T] ziehen, da auch hier kein spezifischer Einbau in Formononetin erfolgt.

Die in der Tab. 2 in Klammern angegebenen Werte wurden mit Formononetin erhalten, das ursprünglich in der Pflanze als Glykosid vorlag. Die Aktivität des Glykosids war durchweg geringer als die des Aglykons *.

Die Versuche mit der 3-fach markierten $p$-Methoxyzimtsäure bestätigen die oben diskutierten Befunde. Nicht nur der überwiegende Teil des Tritiums, sondern auch das ${ }^{14} \mathrm{C}$ der Methoxylgruppe geht beim Einbau in die Isoflavone verloren. Der Aktivitätsverlust muß also durch eine echte Demethylierung und nicht durch einen evtl. Austausch der H-Atome der Methoxylgruppe bedingt sein.

In Analogie zur Demethylierung in tierischen Systemen ${ }^{12}$ dürfte auch in Pflanzen die Demethylierung über Formaldehyd verlaufen. Dadurch tritt bei der Demethylierung ein Tritiumsverlust ein, und das $\mathrm{T} /{ }^{14} \mathrm{C}$-Verhältnis im $\mathrm{C}_{1}$-pool der Pflanze muß kleiner sein als dasjenige in der Methylgruppe. Tatsächlich sinkt das $\mathrm{T} /{ }^{14} \mathrm{C}$-Methyl-Verhältnis in beiden Isoflavonen stark ab (Abb. 3), so daß der Einbau des Methyl $-{ }^{14} \mathrm{C}$ und des $\mathrm{T}$ hauptsächlich durch Wie-

* Bei dem Aglykon handelt es sich wahrscheinlich um ein Gemisch von Aglykon und teilweise hydrolysiertem Glykosid, da bei der Aufarbeitung der Aglyka sicher schon ein Teil der Glykoside gespalten wurde.

12 Siehe z. B. Demethylierung von Biochanin A zu Genistein mit Rattenlebermikrosomen: A. Nilsson u. B. C. Johnson, Arch. Biochem. Biophysics 101, 494 [1963]. 
dereinbau aus dem $\mathrm{C}_{1}$-pool zustande kommen muß. Nach den Ergebnissen mit dem dreifach markierten Methoxychalkon wird auch dieses nicht intakt in die Isoflavone eingebaut. Überraschenderweise steigt hier das Verhältnis Methyl $-{ }^{14} \mathrm{C} / \beta-{ }^{14} \mathrm{C}$ innerhalb der ersten 20 Stdn. zunächst stark an, um dann wieder abzufallen (Abb.4). Dies ließe sich durch schnelle Markierung des $\mathrm{C}_{1}$-pools und raschem Wiedereinbau der Aktivität in die Methoxylgruppe erklären, während der Einbau des $\beta-{ }^{14} \mathrm{C}$ wegen der geringen Spezifität des Einbaus in Formononetin im Vergleich zu Biochanin A wahrscheinlich zum größten Teil über Abbauprodukte ${ }^{13}$ erfolgt und langsamer verläuft.

Durch die schnelle Demethylierung der eingesetzten Vorstufen ist daher eine Aussage, auf welcher Stufe der Bioynthese die Methylierung stattfindet, nicht möglich. Zur Klärung dieser Frage sind Versuche zur Methylierung und Demethylierung in zellfreien Systemen notwendig. Immerhin zeigt der sehr gute Einbau des Trihydroxychalkons in Formononetin, daß die Methylierung auf einer relativ späten Stufe erfolgen kann.

\section{Experimenteller Teil}

Markierte Verbindungen

Zimtsäure- $\left[3-{ }^{14} \mathrm{C}\right]{ }^{14}, \quad p$-Hydroxyzimtsäure- $\left[3-{ }^{14} \mathrm{C}\right]{ }^{15}$ und $2^{\prime} \cdot 4^{\prime} .4$-Trihydroxychalkon- $\left[\beta \cdot{ }^{14} \mathrm{C}\right]{ }^{6,16}$ wurden nach bereits veröffentlichten Vorschriften synthetisiert. Methyljodid- $\left[{ }^{14} \mathrm{C}\right]$ bezogen wir vom Radiochemical Center Amersham.

$$
\text { p-Methoxyzimtsäure- }\left[3-{ }^{14} \mathrm{C}\right]
$$

$18,8 \mathrm{mg}$ p-Cumarsäure- $\left[3{ }^{-14} \mathrm{C}\right]$ wurden in $3 \mathrm{~cm}^{3}$ abs. Aceton mit $100 \mathrm{mg}$ trockenem $\mathrm{K}_{2} \mathrm{CO}_{3}$ und $0,16 \mathrm{~cm}^{3} \mathrm{Di}$ methylsulfat 5 Stdn. unter Rühren am Rückfluß zum Sieden erhitzt. Nach Filtration wurde das Aceton verdampft und der rohe $p$-Methoxyzimtsäuremethylester auf mit Methanol/konz. HCl (95:5) vorgewaschenen Kieselgelplatten mit Benzol/Essigsäure/Wasser (6:7:3) gereinigt. Der Ester wurde sodann mit $5 \mathrm{~cm}^{3}$ 10-proz. methanolischer KOH 6 Stdn. unter Stickstoff und Rückfluß zum Sieden erhitzt. Nach Abziehen des Methanols i. Vak. nahm man mit $8 \mathrm{~cm}^{3}$ Wasser auf, filtrierte und fällte die Säure mit $\mathrm{HCl}$. Ausbeute: $11,22 \mathrm{mg}$ (55\%). Spez. Aktivität $1,466 \mathrm{mC} / \mathrm{mMol}$.

13 L. Patschke, D. Hess u. H. Grisebach, Z. Naturforschg. 19 b, 1114 [1964].

14 S. H. Brown u. A. C. Neish, Canad. J. Biochem. Physiol. 33, 948 [1955] ; H. C. Brown u. B. C. Subba Rao, J. Amer. chem. Soc. 80, 5377 [1958].

\section{4-Methoxy-2' $\cdot 4^{\prime}-$ Dihydroxychalkon- $\left[\beta-{ }^{14} C\right]$}

$51,8 \mathrm{mg} p$-Hydroxybenzaldehyd -[carbonyl $\left.-{ }^{14} \mathrm{C}\right]{ }^{6}$ wurden in $3 \mathrm{~cm}^{3}$ abs. Aceton mit $200 \mathrm{mg}$ trockenem $\mathrm{K}_{2} \mathrm{CO}_{3}$ und $1,5 \mathrm{~cm}^{3}$ Methyljodid unter Stickstoff und Rühren am Rückfluß zum Sieden erhitzt. Der Fortgang der Methylierung wurde dünnschichtchromatographisch auf Kieselgel mit Benzol/Methanol (95:5) verfolgt (Sprühreagens Dinitrophenylhydrazin). Nach $6 \mathrm{Stdn}$. wurde vom $\mathrm{K}_{2} \mathrm{CO}_{3}$ filtriert und das Aceton aus einem Kältebad vorsichtig i. Vak. entfernt. Den Aldehyd nahm man mit peroxidfreiem Äther auf und sublimierte nach Abziehen des Äthers bei $0,2 \mathrm{~mm} \mathrm{Hg}$ und einer Ölbadtemperatur von $70^{\circ} \mathrm{C}$. Der farblose kristalline Anisaldehyd-[carbonyl- ${ }^{14} \mathrm{C}$ ] wurde vom Kühlfinger mit $1 \mathrm{~cm}^{3}$ Äthanol abgespült und zu einer warmen Lösung von $220 \mathrm{mg}$ Resacetophenon-4-glucosidtetra-acetat ${ }^{7}$ in $2 \mathrm{~cm}^{3}$ 60-proz. KOH gegeben. Nach weiterer Zugabe von $0,6 \mathrm{~cm}^{3} \mathrm{KOH}$ wurde $15 \mathrm{Min}$. unter Rühren und Stickstoff auf $70^{\circ} \mathrm{C}$ erhitzt. Danach ließ man 17 Stdn. bei Raumtemperatur stehen und säuerte mit 15-proz. HCl unter Kühlung an. Ausbeute: $156 \mathrm{mg}$ 4-Methoxy-2'-hydroxy-4'-glucosidochalkon- $\left[\beta-{ }^{14} \mathrm{C}\right]$.

$72,82 \mathrm{mg}$ des rohen Glucosids wurden in $5 \mathrm{~cm}^{3}$ Äthanol und $5 \mathrm{~cm}^{3} 2-n$. HCl 1,5 Stdn. unter Stickstoff und Rückfluß zum Sieden erhitzt. Nach Entfernen des Alkohols i. Vak. wurde das Aglykon aus der wäßrigen Lösung mit Äther ausgeschüttelt. Zur Reinigung chromatographierte man auf Kieselgelplatten mit Toluol/ Ameisensäureäthylester/Ameisensäure $(5: 4: 1)$ und auf Whatman 3 MM-Papier mit 30-proz. Essigsäure. Spez. Aktivität: $3,09 \mathrm{mC} / \mathrm{mMol}$.

\section{Daidzein-[T]}

$61 \mathrm{mg}$ Daidzein wurden nach einer modifizierten Wilzbach-Methode ${ }^{17}$ vom 4. 10.-4. 11. $1965 \mathrm{mit}$ 10 Curie T-Gas inkubiert. Das Daidzein wurde mit siedendem Äthanol/Aceton von den Glaskugeln entfernt und 3-mal aus äthanolischer Lösung i. Vak. zur Trockne gebracht. Die weiteren Reinigungsschritte sind in der Tab. 5 zusammengestellt. Spez. Aktivität $18,34 \mathrm{mC} /$ $\mathrm{mMol}$.

\begin{tabular}{lc}
\hline 3-mal kristallisiert aus Aceton/Wasser bzw. & ipm/mMol \\
Methanol/Wasser & {$\left[\cdot 10^{-10}\right]$} \\
DS mit Benzol/Methanol/Essigsäure/Wasser & \\
(72:13:6,5:8) & 3,22 \\
DS mit Chloroform/Isopropanol (10:1) & 2,118 \\
DS mit Benzol/Methanol (25:5) & 2,124 \\
Verdünnungsanalyse nach Kristallisation & \\
aus Äthanol & 2,23 \\
Papierchromatogramm mit 30-proz. Essig- & \\
säure & 2,036 \\
\hline
\end{tabular}

Tab. 5. Reinigung des Daidzein-[T] nach $\mathrm{W} i \mathrm{lzb}$ a ch . Markierung. DS = Dünnschichtchromatographie auf Kieselgel.

15 K. O. Vollmer u. H. Grisebach, Z. Naturforschg. 21 b, 435 [1966].

16 H. Grisebach u. L. Patschie, Chem. Ber. 93, 2326 [1960].

17 H. Wollenberg u. M. Wenzel, Z. Naturforschg. 18 b, 8 [1963]. Herrn Dr. P. E. Schulze (Schering AG, Berlin) danken wir vielmals für die Tritierung. 


$$
\text { p-Methoxyzimtsäure-[methyl-T] }
$$

$50 \mathrm{mg} p$-Cumarsäuremethylester wurden in $5 \mathrm{~cm}^{3}$ abs. Aceton mit $300 \mathrm{mg}$ trockenem $\mathrm{K}_{2} \mathrm{CO}_{3}$ und $0,13 \mathrm{~cm}^{3}$ Methyljodid- $[\mathrm{T}]^{9} 3,5$ Stdn. unter Rückfluß und Rühren zum Sieden erhitzt. Die Methylierung wurde dünnschichtchromatographisch auf Kieselgelplatten mit dem Laufmittel Benzol/Methanol (95:5) verfolgt. Die Aufarbeitung erfolgte wie bei der p-Methoxyzimtsäure$\left[3{ }^{14} \mathrm{C}\right]$. Den gereinigten Ester verseifte man mit $15 \mathrm{~cm}^{3}$ 10-proz. methanolischer $\mathrm{KOH}$. Zur Reinigung wurde die Säure auf Whatman 3 MM-Papier mit 15-proz. Essigsäure chromatographiert. Ausbeute: $36,2 \mathrm{mg}$. Spez. Aktivität $3,04 \mathrm{mC} / \mathrm{mMol}$.

\section{4-Methoxy-2'-4'-Dihydroxychalkon-[methyl-T]}

$58,4 \mathrm{mg} p$-Hydroxy-benzaldehyd wurden unter den bei dem ${ }^{14} \mathrm{C}$-markierten Chalkon geschilderten Bedingungen mit insgesamt $0,102 \mathrm{~cm}^{3}$ Methyljodid[T] zur Reaktion gebracht. Die Kondensation des Anisaldehyds zum Chalkon und dessen Reinigung erfolgte wie oben beschrieben. Spez. Aktivität $3,06 \mathrm{mC} / \mathrm{mMol}$.

$$
\begin{gathered}
\text { p-Methoxyzimtsäure-[methyl- } \left.{ }^{14} \mathrm{C}\right], 4-M e t h o x y-2^{\prime} .4^{\prime} \text { - } \\
\text { Dihydroxychalkon- }\left[\text { methyl }-{ }^{14} \mathrm{C}\right]
\end{gathered}
$$

Beide Verbindungen wurden nach den oben für die T-haltigen Verbindungen angegebenen Methoden mit Methyljodid- $\left[{ }^{14} \mathrm{C}\right]$ synthetisiert. Spez. Aktivität der Zimtsäure $0,46 \mathrm{mC} / \mathrm{mMol}$, des Chalkons $0,602 \mathrm{mC} /$ mMol.

Untersuchungen über die besten Bedingungenzur Aufnahmedes 4-Meth. oxy-2'.4'-Dihydroxychalkons in die

$$
\text { Pflanze }
$$

Bietet man das Chalkon als Mononatriumsalz mit einem 20-proz. Überschuß von Alkali an, so fällt wegen der von den angeschnittenen Pflanzen ausgeschie-

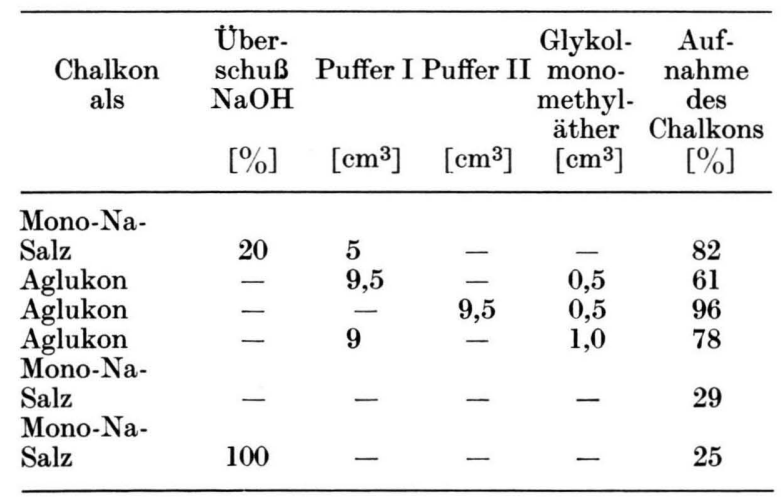

Tab. 6. Aufnahme des 4-Methoxy-2'.4'-dihydroxychalkons in Cicer-Pflanzen ohne Wurzeln unter verschiedenen Bedingungen. Puffer $\mathrm{I}=\mathrm{NaH}_{2} \mathrm{PO}_{4} / \mathrm{Na}_{2} \mathrm{HPO}_{4}, 0,05-m$., $p_{\mathrm{H}} 8,5$. Puffer II $=\mathrm{Na}_{4} \mathrm{P}_{2} \mathrm{O}_{7} / \mathrm{HCl}, 0,1-m ., p_{\mathrm{H}} 8,5$. denen Säuren das Chalkon alsbald wieder aus. Die in der Tab. 6 zusammengestellten Versuche wurden jeweils mit 15 Cicerpflanzen ohne Wurzeln in feuchter Atmosphäre durchgeführt. Das Volumen der Lösung, welche $1 \mathrm{mg}$ Chalkon enthielt, betrug jeweils $10 \mathrm{~cm}^{3}$. Dauer der Versuche 31 Stunden. Die verbliebenen Chalkonmengen wurden nach Extraktion mit Äther in Methanol im UV bestimmt.

Kinetik des Einbaus der dreifach markierten $p$-Methoxyzimtsäure und des dreifach markierten Methoxychalkons

Die dreifach markierten Verbindungen wurden durch Mischen aus den einfach markierten hergestellt. Bei den Ansätzen wurden jeweils 2,5 $\mu \mathrm{Mol}$ markierte Verbindung in $0,3 \mathrm{~cm}^{3}$ Glykolmonomethyläther gelöst und sodann 7,7 $\mathrm{cm}^{3}$ Pyrophosphatpuffer (0,1-m., $p_{\mathrm{H}}$ 8,5) hinzugegeben, so daß die Molarität der Lösungen an aktivem Substrat $3,125 \cdot 10^{-4}$ betrug. Pro Versuch wurden 15 gleiche Pflanzen ohne Wurzeln in feuchter Atmosphäre verwendet. Die Parallelversuche liefen $8^{1 / 2}, 18$ und 26 Stunden.

Zur Aufarbeitung spülte man die Pflanzen ab und zerkleinerte sie im Starmix unter Zusatz von $300 \mathrm{~cm}^{3}$ Wasser. Nach 2-stdg. Stehen wurde abgesaugt und die Pflanzenteile gefriergetrocknet. Nach Extraktion mit Methanol, Entfetten mit Petroläther und Trocknen an der Luft versetzte man mit wenig Wasser und ätherte erschöpfend aus. Das Wasser der enzymatischen Hydrolyse wurde ebenfalls ausgeäthert. Die vereinigten Ätherfraktionen chromatographierte man auf Kieselgel mit Benzol/Äthanol (92:8). Formononetin wurde noch einmal mit Chloroform/Isopropanol $(10: 1)$ und Biochanin A mit Äther/Petroläther $(7: 3)$ gereinigt. Nach Verdünnung mit inaktivem Material kristallisierte man einmal aus Methanol und nochmals aus Methanol/Wasser. Dann wurden alle Proben permethyliert und kristallisiert. Đie drei Biochanin A-dimethyläther aus den Versuchen mit dem Methoxychalkon reinigte man nochmals über Kieselgel mit Benzol/Essigester/Methanol/ Petroläther $(6: 4: 1: 2)$ und kristallisierte aus Benzol/Petroläther um.

$$
\text { Messung der Radioaktivität }
$$

Die Messung der Radioaktivität erfolgte in der Gasphase nach Simon und Mitarbeiter ${ }^{18}$. Meßgerät Uni ZS mit Antikoinzidenzanlage von Prof. Berthold, Wildbad/Schwarzwald.

Dem Fonds der Chemischen Industrie und der Badischen Anilin- und Soda. fabrik danken wir für Sachbeihilfen. Fräulein E. LoBsien danken wir für geschickte Mithilfe.

18 H. Simon, H. Daniel u. I. F. Klebe, Angew. Chem. 71, 303 [1959]. 